\title{
IX.
}

Aus dem pharmakol. Institut von Prof. v. Sehroeder in Heidelberg.

\section{Ueber Xanthinkörper im Harn des Leukämikers.}

$$
\text { Von }
$$

Dr. St. Bondsyński und Dr. R. Gottlieb.

Das Schicksal der Xanthinkörper im Organismus steht in naher Beziehung zu der Frage der Harnsäurebildung. Seitdem die nahe Verwandtschaft der Harnsäure zu den Xanthinbasen durch die chemische Forschung festgestellt ist und seitdem die Arbeiten Kossel's and seiner Schule die Entstehung der Xanthinbasen als Zerfallsproducte des Zellkerns erwiesen haben, hat man in ihnen die Quelle der Harnsäure im Organismus gesucht. Ein Beweis dieser Umwandlung konnte aber bisher nicht erbracht werden. Denn nur im Organismus der Vögel, in welchem die Harnsäure bekanntlich als Endproduet des Stoffwechsels eine ganz andere Rolle spielt als beim Säugethiere, hat v. Mach ${ }^{1}$ im Anschluss an die bekannten Versuche Mink ow ski's den Uebergang von Hypoxanthin in Harnsäure nachweisen können. Die Ergebnisse der zahlreichen Versuche am Sängethiere sind dagegen von der ersten umfassenden Arbeit Stadthagen ${ }^{2}{ }^{2}$ ) an bis in die neueste Zeit unklar und vieldeutig geblieben. Immerbin mehren sich aber in den letzten Jahren die Beobachtungen, welche die Harnsäurebildung aus den Xanthinkörpern der Nuclëne wahrscheinlich machen. In dieser Richtung sind vor Allem die Untersuchungen Horbaczewski's ${ }^{3}$ ) zu nennen, der den höchst anffallenden Parallelismus zwischen Harnsäureausscheidung und Leukocytenzahl näher verfolgte und auf Grund dieses Parallelismus die Harnsäure in erster Linie aus den Nuclë̈nen dieser vergänglichsten Zellen, der Leukocyten, herleitet. Weiter ist es aber Horbaczewski auch ge-

1) Archiv f. exp. Path. u. Pharm. Bd. XXIV. S. 389.

2) Virchow's Archiv. Bd. CIX. S. 390. 1887.

3) Sitzungsber. d. Wiener Akad. d. W. Bd. C. III. S. 13.1891. 
lungen, durch Digestion von Milzbrei mit Blut bei Körperwärme Harnsäure zu erhalten. Ausserbalb des Organismus haben endlich Emil Fischer und $A \mathrm{ch}^{1}$ ) in allerjüngster Zeit durch Ueberfubrung der Dimethylharnsäure in Theophyllin den auch von der synthetischen Chemie lange vergebens gesuchten Uebergang der einen Gruppe in die andere gefunden; hierdurch erscheint die chemische Grundlage für die Annahme einer Umwandlung auch der Xanthinkörper in Harnsäure um so fester begründet.

Der Annahme einer Harnsäurebildung aus Xanthinkörpern scheinen nur die Resultate zu widersprechen, welche die Versuche nach Zufuhr von Xanthinkörpern per os ergeben haben; dieselben weisen nämlich eher auf eine leichte Zerstörbarkeit im Organismus als auf einen Uebergang in Harnsäure hin. So haben Nencki und Siebe ${ }^{2}$ ) nach Verfütterung von Xanthin, Kerner ${ }^{3}$ ) und St ad thage ${ }^{4}$ ) nach Guanin an Hunden weder Harnsäurevermehrung noch auch eine unveränderte Ausscheidung der verfïtterten Substanzen beobachtet. Die Versuche mit Darreichung von Nucleïn, der Muttersubstanz der Xanthinkörper ergaben weniger übereinstimmende Resultate. Stadthagen (l. c.) und $\mathrm{Gumlich}{ }^{5}$ ) kamen zu negativen Ergebnissen, während Horbaczewski (l. c.) und Richter ${ }^{6}$ ) nach Einnahme von Nucleïn und nucleïnsaurem Natron Steigerung der Harnsäureausscheidung constatirten; da aber die Nucleïnsäure gleichzeitig Leukocytose und dadurch vermehrten Untergang von Leukocyten hervorruft, so sind die Ergebnisse keineswegs eindeutig. Die per os eingeführten Xanthinkörper werden jedenfalls im gesunden Organismus leicht weiter, wohl bis zu den nächsten Vorstufen des Harnstoffs zerstört.

Bei diesem Stande der Frage nach der Harnsäurebildung aus Xanthinbasen erlangt das Studium eines pathologischen Processes um so grössere Bedeutung, in welchem neben Harnsäure auch die Xanthinkörper in vermehrter Menge im Harn auftreten. Ein Fall von Leukämie, der sehr erhebliche Mengen von Xanthinbasen ausschied und den Herr Geheimrath Erb die Güte hatte uns zur Untersuchung zu überlassen, bot uns eine willkommene Gelegenheit, einen Beitrag zur Kenntniss der Ausscheidung dieser Substanzen zu liefern.

1) Berichte d. Berliner Akad. 1895.

2) Pflüger's Archiv. Bd. XXXI. S. 347. $18 \$ 3$.

3) Annal. d. Chem. Bd. CIII. S. 249.

4) Virchow's Archiv. Bd. CIX. S. 417. 1887.

5) Zeitschr. f. physiol. Chemie. Bd. XVIII. S. 508.

6) Zeitschr. f. klin. Med. Bd. XXVII. S. 290. 
Die folgenden Notizen aus der Krankengeschichte des Falles verdanken wir der Freundlichkeit des Herrn Assistenzarzt Dr. Battlehner.

Pat. F. F., der im Anschluss an Gelenkrheumatismus seit mehreren Jahren an leichteren Störungen seitens des Herzens leidet, bemerkte zuerst vor etwa 2 Jahren Drüsenschwellungen in der Achsel und Leistengegend. Bei der Aufnahme (6. Dec. 1894) fand sich eine sehr bedeutende Anschwellung der Unterkieferdrüsen sowie der Cervicaldrüsen, die dicke Drüsenpackete bilden; die Achseldrïsen sind bis Gänseeigrösse, die Leistendrüsen bis Kastaniengrösse angeschwollen. Herzdämpfung etwas vergrössert, über dem Herzen sykolisches Geräusch. Abdomen stark aufgetrieben. Ein grosser harter und glatter Tumor der Milz von $33 \mathrm{~cm}$ Länge und $17 \mathrm{~cm}$ Breite. Etwas Oedem an den Füssen; Dyspnoe besteht nicht. B l utbe fund: Hämoglobingehalt (F eis c hl) 30 Proc. Zahl der rothen Blutkörperchen im Cubikcentimeter 1500000 , die der weissen 500000 . Verhältniss der rothen zu den weissen Blutzellen 1:3. Die weissen Blutzellen sind hauptsächlich Lymphocyten, die Anzahl grosser Leukocyten myelogenen Ursprungs ist gering. Diagnose: Lymphatischlienale und wahrscheinlich auch myelogene Leukämie.

Verschiedene Blutuntersuchungen während der Behandlung ergaben immer das Verhältniss der rothen Blutkörperchen za den weissen $1: 3$, während die Anzahl der rothen und der Hämoglobingehalt etwas anstiegen, dabei nahm die Anzahl eosinophiler Leukocyten myelogenen Ursprungs während der Beobachtung zu. Im späteren Verlaufe der Krankheit stellte sich eine ausgedehnte Eiterung am rechten Unterkiefer ein, die zur Nekrose des Unterkiefers fuhrte und die Resection desselben nöthig machte, nach der (12. März) der Exitus eintrat. Anatomische Diagnose: Lymphatisch-lienale und myelogene Leukämie.

Bei der Harnuntersuchung wandten wir unser Interesse in erster Linie der Ausscheidung der Xanthinkörper zu. Dass dieselben bei Leukämie vermehrt erscheinen, ist seit langer Zeit bekannt. Insbesondere wurde ihre Zunahme von Scherer ${ }^{1}$, Salomon ${ }^{2}$ ) und Kossel ${ }^{3}$ ) u. A. nachgewiesen; Stadthagen (l. c.) versuchte dann zuerst einen quantitativen Vergleich der Xanthinbasen in normalem und Leukämieharn and fand die tägliche normale Ausscheidung (von $3 \mathrm{cg}$ ) auf mehr als das Doppelte $(8 \mathrm{cg})$ gesteigert; doch sind die absoluten Werthe, die er durch Silberfällung ermittelte, sehr gering. In neuerer Zeit hat dann Drechsel ${ }^{4}$ ) auf die Fällung der Harnsäure und der Xanthinbasen durch Kupferoxydul aufmerksam gemacht und $\mathrm{Kr}$ ü ge ${ }^{5}$ ) dies Verfahren in zweckmässiger Weise durch

1) Verhandl. d. Würzburger med. Ges. II. 1852.

2) Zeitschr. f. physiol. Chemie. Bd. II. S. 80.

3) Ebenda. Bd. VII. S. 22.

4) Bericht d. deutsch. chem. Ges. Bd. XXV. S. 2454.

5) Zeitschr. f. physiol. Chemie. Bd. XVIII. S. 351.

Arch i f f. experiment. Pathol, u. Pharmakol. XXXVI. Bd. 
gleichzeitige Anwendung von Kupfersulfat und Natriumbisulfit als Reductionsmittel modificirt. Indem $\mathrm{Kr}$ üger zeigte, dass die Ausfällung der Harnsäure und der normalen Xanthinbasen des Harns in der Wärme eine vollständige sei, konnte er eine Methode ihrer quantitativen Bestimmung ausarbeiten; indem einerseits der $\mathrm{N}$-Gehait des abgeschiedenen Kupferoxydulniedersehlags nach $\mathrm{K}$ jeldahl bestimmt wird, in einer anderen Harnportion aber der $\mathrm{N}$-Gehalt der nach Salkowski-Ludwig abgeschiedenen Harnsäure, ergiebt sich aus der Differenz der in den Xanthinkörpern des Harns ausgeschiedene N. Schon Krüger ${ }^{1}$ ) hat mittelst dieser Methode eine Untersuchungsreihe an einem Leukämiker ausgefïhrt.

Auch wir wandten die $\mathrm{Kr}$ ïg er'sche Methode zur Bestimmung der Xanthinkörper an. Wir bestimmten ferner täglich den Gesammtstickstoff nach Kjeldahl, die Harnsäure nach Salkowski-Ludwig, endlich den Antheil des Harnstickstoffs, der durch Phosphorwolframsäure fällbar ausgeschieden wurde. Die Ergebnisse von zwei Untersuchungsperioden, deren eine 6 Tage und die zweite 9 Tage umfasst, sind in der nebenstehenden Tabelle zusammengestellt.

$\mathrm{Um}$ eine möglichst vollständige Untersuchung des Harns zu geben, wurden ferner an 2 Tagen die Menge der pro die ausgeschiedenen Fettsäuren bestimmt. Wir bedienten uns dabei einer Methode, die sich dem Einen von uns schon in anderweitigen Untersuchungen ${ }^{2}$ ) bewährt hatte. $1000 \mathrm{ccm}$ Harn werden, wie es v. Jaksch${ }^{3}$ ) empfohlen hat, mit circa $100 \mathrm{cem}$ Phosphorsäure von der Dichte 1,275 unter öfterem Zufügen von Wasser so lange destillirt, als das Destillat noch sauer iibergeht. ${ }^{4}$ ) Das Destillat von etwa 2 Liter wird mit Barythydrat alkalisch gemacht, der überschüssige Baryt mit $\mathrm{CO}_{z}$ ausgefällt und eingedampft; der Rückstand wird dann mit wenig Wasser aufgenommen, von einer geringen Menge Baryumearbonat in eine Platinschale abfiltrirt, mit wenig Wasser nachgewaschen, getrocknet und die Barytsalze der Fettsäuren gewogen. Um zu einem Urtheile zu gelangen, welche Fettsäuren in dem so erhaltenen Gemisch der Barytsalze vorwiegen, wurde die Barytbestimmung gemacht. Doch hat die Methode den Vortheil, dass aus dem Rïckstand auch mittelst Alkohol die einzelnen Fettsäuren dargestellt werden können.

1) Deutsche med. Wochenschr. Nr. 33. 1894.

2) Vgl. J. Léva, Virchow's Archiv. Bd. CXXV. S. 73 (Chemischer Bericht).

3) Zeitschr. f. physiol. Chemie. Bd. X.

4) Man muss darauf achten, dass der Inhalt des Kolbens nicht unter $300-$ $400 \mathrm{ccm}$ sinkt, da sonst der Uebergang von Salzsäure zu befürchten ist. In den Versuchen wurde stets auf etwa übergegangene Salzsäure geprüft. 
Ueber Xanthinkörper im Harn des Leukämikers.

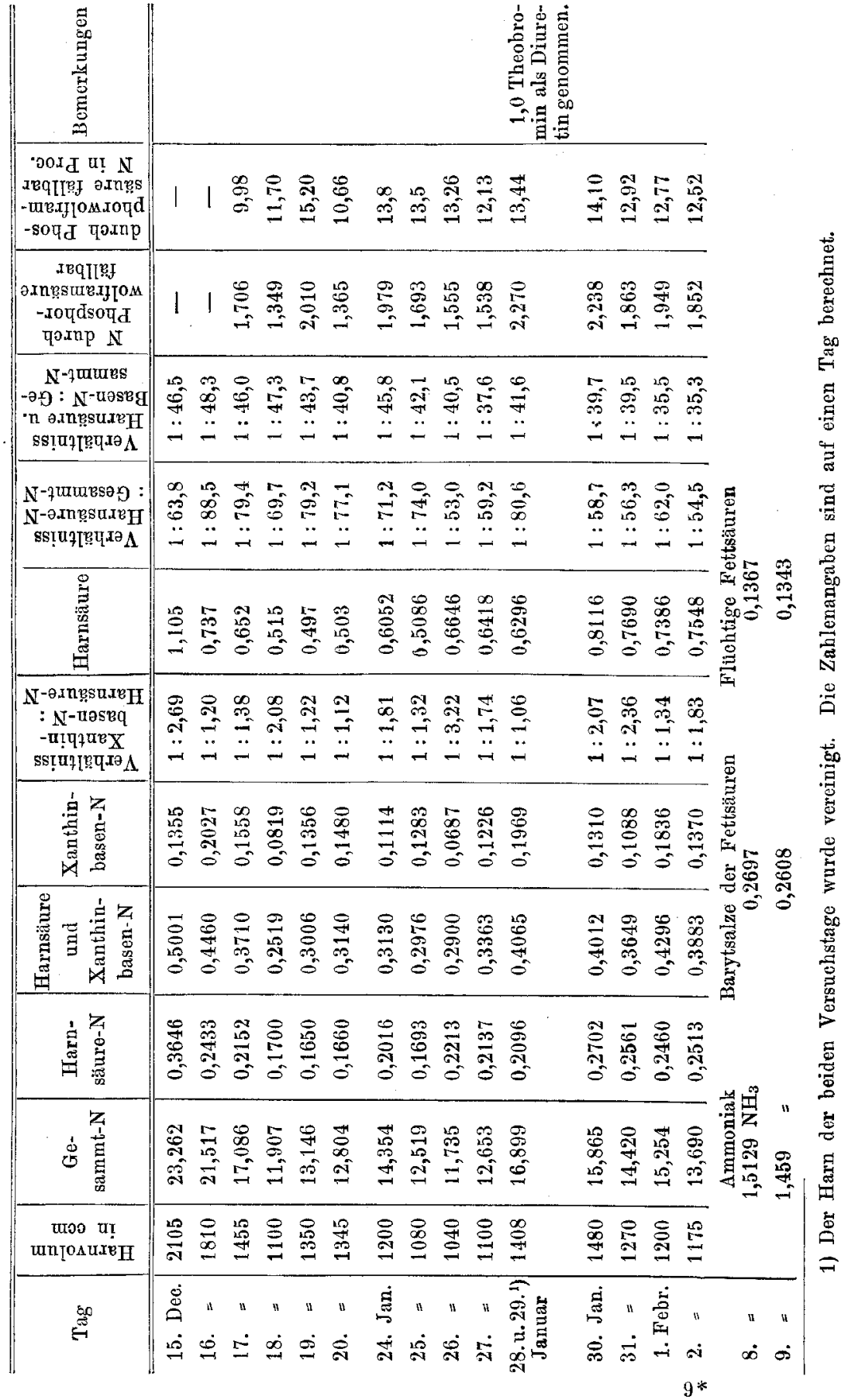


Die Xanthinbasen sind im vorliegenden Falle dauerud und sebr auffallend vermehrt. Da, wie oben erwähnt, die Untersucher vor Kr ü g er nur eine Darstellung der Xanthinkörper aus Leukämieharn, nicht aber ihre quantitative Abscheidung erreichten, so mïssen unsere Resultate vor Allem mit denen $\mathrm{K} r$ iu ger's verglichen werden. Die Ausscheidung der Xanthinkörper ïbertrifft aber im vorliegenden Falle noch erheblich die von $\mathrm{Kr}$ ï ger beobachtete Steigerung. Während in $\mathrm{Kr}$ ru ger's Falle das Verhältniss von Basen-N : Harnsäure-N sich in den Grenzen 1:5 bis 1:3 bewegt, erreicht hier die Ausscheidung der Xanthinbasen bäufig fast den Werth der Harnsäureausscheidung. Gegenuiber einem Mittel von $0,0778 \mathrm{~g}$ Basen-N in der normalen Periode von $\mathrm{Kr}$ ù ge r's Fall, erbielten wir an einzelnen Tagen Werthe von $0,2027 \mathrm{~g}(17$. December) und $0,1836 \mathrm{~g}$ (31. Januar) Basen- $\mathrm{N}$, die 0,5-0,6 $\mathrm{g}$ Xanthinbasen pro die entsprechen würden. Da Krüger als Mittelzahl aus 19 normalen Harnen eine tägliche Ausscheidung von $0,0481 \mathrm{~g} \mathrm{~N}$ in Form der Xanthinbasen bestimmte, so liegt eine Steigerung der Xanthinkörper bis auf das Dreifache und Vierfache ibres normalen Werthes vor. ${ }^{1}$ )

Bei einer Zunahme der Xanthinkörper im Harn, die hier etwa der normalen Harnsäureausscheidung an Menge gleichkommt, erhebt sich die Frage, ob im Organismus des Leukämikers die Fähigkeit beeinträchtigt ist, die Xanthinkörper aus der Nahrung in normaler Weise zu zerstören. Die oben erwähnten Untersuchungen haben für den normalen Organismus die leichte Zerstörbarkeit der per os eingeführten Xanthinkörper ergeben. Wir versuchten deshalb der Frage experimentell näher zu treten, $o b$ diese normale Zerstörung der Xanthinkörper im Organismus des Leukämikers behindert ist. Wir wählten aber zu unseren Versuchen nicht das Xanthin selbst, weil uns dasselbe in völliger Reinheit nicht zur Verfügung stand, dann aber auch wegen der ungeniugenden Kenntniss, die wir für den menschlichen Organismus von seinen Schicksalen besitzen. Dagegen sind jetzt die Schicksale zweier methylirter Derivate des Xanthins, des Theobromin und Coffeïn genauer verfolgt worden. Insbesondere hat im hiesigen Institute Herr cand. med. E. Rost durch genaue quantitative Bestimmungen ermittelt, welcher Antheil von diesen Xanthinderivaten unverändert im Harn wieder-

1) Bei so grossen Xanthinmengen im Harne kann wohl heute kaum mehr an die Erklärung ihres Auftretens bei Leukämie gedacht werden, welche $\mathbf{S}$ tad $\mathrm{t}$ hagen angedeutet hat, dass sie etwa auf das Vorkommen lymphatischer Neubildungen in den Nieren and vermehrter Abstossung des Nierenepithels zurückzuführen wären. 
erscheint. Für Theobromin fand er beim Menschen eine unveränderte Ausscheidung von 13-20 Proc. Ferner gelang es uns nach Einnahme von Coffeïn und Theobromin als intermediäres Stoffwechselproduct Methylxanthin darzustellen und dadurch den Abbau der methylirten Xanthinderivate durch Abspaltung der Methylgruppe zu erweisen. Eine vermehrte Ausscheidung von Xanthin liess sich aber nach Einnahme der methylirten Derivate nicht nachweisen; dasselbe wird demnach im normalen Organismus wohl leicht weiter zerstört. Coffeïn und Theobromin durcblaufen also bei ihrer Zerstörung höchst wahrscheinlich die Stufe des Xanthins und so empfahl es sich zum Studium des Verhaltens der Xanthinkörper im Organismus des Lenkämikers, diese in ihren Geschicken genauer bekannten Verbindungen heranzuziehen. Unser Patient erhielt in zwei Versuchen $1 \mathrm{~g}$ und 2,5 Theobromin in Form von Diuretin. Der Harn des Versuchstages und des darauffolgenden Tages wurden vereint und 1500 und $2000 \mathrm{ccm}$ davon in der von E. Rost beschriebenen Weise auf Theobromin verarbeitet; nebenbei bestimmten wir auch die in das Chloroformextract der zerlegten Phosphorwolframsäurefällung übergegangene Menge Methylxanthin. Wir erhielten in dem einen Versuche 12 Proc. des eingenommenen Theobromin und 28 Proc. Methylxanthin, in dem zweiten Versuche 15 Proc. Theobromin wieder. In einem Controlversuche überzeugten wir uns, dass aus der gleichen Menge desselben Harns ohne vorherige Darreichung von Theobromin nur minimale Mengen von Xanthinkörpern bei der gleichen Behandlung in das Chloroformextract ïbergehen.

Es geht aus den Versuchen hervor, dass das per os genommene Xanthinderivat, Theobromin im Organismus des Leukämikers ebenso vollständig zerstört wird wie bei Gesunden; es erscheint ebenso viel unverändert und als Metbylxanthin wieder und eine merkliche Zunahme der Xanthinkörper ist nicht nachweisbar. ${ }^{1)}$ Eine Stoffwechselanomalie, durch welche die Zerstörung der Xanthinkörper im Organismus behindert würde, liegt demnach in der Leukämie nicht vor.

Hält man nun die Thatsache der normalen Zerstörbarkeit der

1) Die z. B. in dem ersten Theobrominversuch (28. und 29. Januar der Tabelle) auftretende Steigerung des Xanthinbasen- $\mathrm{N}$ erklärt sich aus dem Auftreten von Methylxanthin, das sich bei der Bestimmung wie die Xanthinbasen verhält. Ein Vergleich der Steigerung von $0,074 \mathrm{~g} \mathrm{~N}$ mit der $\mathrm{N}$-Menge, die sich aus dem gleichzeitig bestimmten Methylxanthin $(6,2826 \mathrm{~g})$ berechnet, zeigt, dass jedenfalls keine nennenswerthe Zunahme des Basen- $N$ auf Rechnung der anderen Xanthinkörper zu setzen ist. 
per os eingefuhrten Xanthinkörper mit der abnormen Steigerung dieser Basen im Harne zusammen, so scheint daraus der Schluss hervorzugehen, dass die aus dem Kernzerfall, speciell dem Kernzerfall der Leukocyten hervorgehenden Xanthinkörper sich im Organismus ganzanders verhalten wie die vom Darmaus resorbirten. Während die letzteren beim Leukämiker in derselben Weise zerstört werden wie im normalen Organismus, treten die aus dem Kernzerfall stammenden in abnormer Menge im Harn auf. Die Xan: thinkörper verhalten sich darin wie die Harnsäure; denn auch von der Harnsäure konnte Stad thagen zeigen, dass sie nach Einnahme per os von dem Leukämiker vollständig zerstört wird und nicht als solche im Harne erscheint. - Die Xanthinkörper im Harn werden demnach nicht infolge behinderter Zerstörung der per os eingeführten vermehrt ausgeschieden, sondern stammen aus dem Organismus selbst.

Zur Beurtheilung der Frage, welche Bedeutung den Xanthinbasen im Harn zukommt, ergeben sich weitere Anhaltspunkte aus dem täglichen Vergleich von Harnsüure- und Xanthinbasenausscheidung. Die Curven beider Ausscheidungen gehen nämlich keineswegs parallel; wenn vielmehr die Menge der ausgeschiedenen Harnsäure über die Norm steigt, sinkt die Menge der Xanthinbasen im Harn und umgekehrt entspricht einem Absinken der Harnsäureausscheidung ein Steigen der im Harn enthaltenen Xanthinkörper. Es erklärt sich dieses Verhalten gewiss am ungezwungensten durch die Annahme, dass die Xanthinbasen an Stelle der Harnsäure ausgeschieden werden und dass die Harnsäure zam Theile wenigstens aus den Xanthinkörpern stammt. In dem von uns untersuchten Falle würde diese angenommene Umwandlung von einem Tage zum anderen nicht unbeträchtlich schwanken, so dass das Verhältniss des pro die ausgeschiedenen Basen- $N$ zum Harnsäure- $N$ sich zwischen $1: 3$ und $1: 1$ bewegt.

Eine solche Vertretung der Harnsäure durch Xanthinkörper spricht sich auch in den relativen Verbältnissen der Harnsäureausscbeidung einerseits und der Summe der Harnsäure und Xanthinbasen andrerseits zur Gesammtzersetzung des Organismus aus. Während nämlich das Verhältniss Harnsäure- $N$ zum Gesammt- $N$ in sehr weiten Grenzen sich bewegt - in der ersten Versuchsperiode z. B. zwischen 1:63 und 1:88 - und dabei unregelmässige und plötzliche Schwankungen aufweist, zeigt das Verhältniss von Harnsäure- + Basen-N zum Gesammt- $N$ eine regelmässige Curve und schwankt in viel engeren Grenzen - in der gleichen Versuchsperiode von $1: 48,3$ bis 
1:40,8. Auch dieser Vergleich spricht zu Gunsten der Annahme, dass man nur die Gesammtsumme von Harnsäure + Xanthinbasen als ein Maass des Nucleïnzerfalls und besonders des Leukocytenstoffwechsels anzusehn hat; deshalb steht der Werth dieser Gesammtsumme in viel constanterem Verhältniss zum ausgeschiedenen Gesammt-N, als die einzelnen Factoren, deren Werthe sich oft sprung. weise ändern.

Es bleibt abzuwarten, ob die chemische Untersuchung anderer Leukämiefälle ähnliche Verhältnisse zwischen der Ausscheidung der Harnsäure- und der Xanthinbasen im Harn ergeben wird. Doch zeigt schon der eine hier näher untersuchte Fall, wie fruchtbringend für die Beurtheilung der Fragen über die Harnsäurebildung sich die Bestimmung der Xanthinbasen erweisen dürfte, deren Ausfuhrung nach der Methode von Krüger keinerlei Schwierigkeiten bietet. Es erscheint so beachtenswerth, dass die so bedeutende Steigerung der Xanthinbasenausscheidung sich in einem Falle von Leukämie findet, bei dem die Harnsäure nur wenig über die Norm vermehrt ist, ja an einzelnen Tagen normale Werthe von 1/70 und 1/80 der Gesammt$\mathrm{N}$-Ausscheidung darbietet. In der Literatur finden sich auch seit der Anwendung der Salkowski-Ludwig'schen Harnsäurebestimmung immer einzelne Fälle von Leukämie, in denen das sonst so constante Sympton der Harnsäuresteigerung fehlt oder sehr gering ist (Salkowski ${ }^{1}$ ), Bohland und Seburz ${ }^{2}$ ), Mathes $\left.{ }^{3}\right)$ ). Diese Fälle bilden eine sehr auffallende Ausnahme ron dem sonst so regelmässigen Parallelismus zwischen Leukocytenzahl und Harnsäureausscheidung, dessen extremsten Fall sonst gerade die Leukämie darstellt. Es darf vielleicht vermuthet werden, dass auch in diesen Fällen an Stelle einer gesteigerten Harnsäureausscheidung eine analoge Vermehrung der Xanthinbasen im Harn vorlag und der Beobachtung entgangen war.

Das Verhalten von Harnsäure- und Xanthinbasenausscheidung: scheint demnach die Annahme zu stützen, dass die Xanthinbasen an Stelle der Harnsäure auftreten und als ihre Vorstufen im Harn bei Leukämie anzusehen sind.

Nencki und Sieber ${ }^{4}$ haben wohl zuerst die Ansicht geäussert, dass die Harnsäure in der Norm durch Oxydation der Xanthinkörper entstehe, die in der Leukämie bei herabgesetzter Oxyda-

1) Virchow's Archiv. Bd. L.

2) Inaug.-Diss. Bonn 1890.

3) Berliner klin. Wochenschr. 1894.

4) Pflüger's Archiv. Bd. XXXI. 
tion unverändert erscheinen. Es entsteht somit die Frage, ob sich im vorliegenden Falle Anhaltspunkte dafür finden, dass eine verminderte Oxydationsfähigkeit der Gewebe die Steigerung der X.anthinbasenausscheidung bedingt. Die ältere Ansicht von den Stoffwechselveränderungen bei Leukämie, dass die verminderte Anzahl der Sauerstoffüberträger eine Herabsetzung der Oxydationen in den Geweben zur Folge habe und deshalb die Harnsäure anf einer niedrigeren Oxydationsstufe stehen bleibe, darf allerdings als längst widerlegt gelten. Denn Pette $\mathrm{nk}$ ofer und Voit ${ }^{1)}$ zeigten bekanntlich, dass die Sauerstoffaufnahme gegenüber dem gesunden Organismus keineswegs herabgesetzt ist. In neuerer Zeit sind dann $\mathrm{Kraus}$ und (hvostek2) and Bohland und Geppertis) zu demselben Resultate gekommen. Wenn demnach auch feststeht, dass die Harnsäurevermehrung in typischen Fällen von Leukämie mit einer Herabsetzung der Oxydationsvorgänge nichts zu thun hat, so könnte doch gerade in jener Minderzahl von Fällen, in denen weniger Harnsäure, aber viel Xanthinbasen ausgeschieden werden, Oxydationshemmung eine Rolle spielen. Dass aber die Oxydation in den Geweben in einzelnen Fällen von Leukämie herabgesetzt sein kann, beweist das Experiment der Benzoldarreichung, welches Nencki und Sieber ${ }^{4}$ ) an einem Leukämiker einen sehr auffallenden Unterschied gegenüber gesunden Versuchspersonen ergab. Sie fanden die Fäbig. keit, Benzol zu Phenol zu oxydiren, bei dem Leukämiekranken ganz enorm herabgesetzt.

Wir waren nicht in der Lage, den respiratorischen Gaswechsel des Patienten zu untersuchen; dass aber im vorliegenden Falle in der That eine Herabsetzung der Oxydationsvorgänge in den Geweben bestand, dafür sprach das Auftreten grosser Mengen von flüchtigen Fettsäuren im Harn, deren Ausscheidung weit über die Norm gesteigert war. Entsprechend dieser vermehrten Säuremenge erreichte auch die Ammoniakausscheidung einen sehr hohen Werth, im Gegensatze zu anderen Fällen von Leukämie, in den Hallervorden ${ }^{5}$ ) und Stadelmann ${ }^{6}$ ) eher niedrige Ammoniakzahlen ermittelten. Der Patient schied am 8. Februar 1,5129 g, am 9. Februar 1,459 g NH aus. Die Bestimmung der flüchtigen Fettsäuren an denselben Tagen

1) Zeitschr. f. Biologie. Bd. V. S. 369.1869.

2) Wiener med. Wochenschr. 1891. Nr. 33.

3) R. Meyer, Inaug.- Diss. Bonn 1892.

4) Journal f. prakt. Chemie. N. F. Bd. XXVI. S. 41.

5) Archiv f. exp. Path. u. Pharm. Bd. XII. S. 274.

6) Archiy f. klin. Med. Bd. XXXIII. S. 536. 
ergab den ungemein hohen Werth von $0,2697 \mathrm{~g}$ und $0,2608 \mathrm{~g} \mathrm{Ba-}$ ryumsalzen in der 24 stündigen Harnmenge. Das Gemisch der Barytsalze wies einen Baryumgehalt von 50,0 Proc. und 49,2 Proc. auf. Nach Abzug des Baryums und Addition des entsprechenden H-Aequivalents berechnet sich aus den Baryumsalzen die Menge der gefundenen Fettsäuren am 8. und 9. Februar anf 0,1367 und $0,1343 \mathrm{~g}$. Es liegt also eine auffallend hohe Steigerung vor gegenüber den geringen Spuren flüchtiger Säuren, die in der Norm vorkommen und die v. Jaksch auf höchstens $0,008 \mathrm{~g}$ pro die bestimmte. Dass eine so grosse Menge der sonst leicht oxydirbaren Säuren der Oxydation entgeht, ist wohl nur durch die Annahme einer herabgesetzten Oxydation in dem beschriebenen Leukämiefalle zu deuten und es liegt nahe, auch die Vermehrung des Xanthinkörpers im Harn mit diesem Symptome in Beziehung zu bringen.

Wenn sich aber auch im Gesammthaushalte des Organismus kein Symptom verminderten Oxydationsvermögens nachweisen liesse und zur Erklärung der abnormen Vermehrung der Xanthinkörper herangezogen werden könnte, so wäre es doch sehr wohl denkbar, dass gerade in jenen Zellen die Oxydationsvorgänge bei Leukämie gestört wären, in denen sonst die angenommene Umwandlung der Xanthinkörper in Harnsäure vor sich geht. Vom Standpunkte der Horbaczewski'schen Theorie liegt es nahe, dabei an die Leakocyten zu denken. Die gesteigerte Harnsäureausscheidung bei Leukämie ist nach dieser Theorie als Ausdruck für die pathologische Vermehrung der Leukocytenzahl anzusehn, die Ausscheidung der Xanthinkörper liesse sich aus einer Störung im Ablaufe der Zellthätigkeit der Leukocyten erklären.

Zum Schlusse erlauben wir uns Hern Geheimrath Erb für die freundliche Ueberlassung des Materials unseren besten Dank auszusprechen.

Heidelberg, Mai 1895. 